\title{
Dietary determinants of hepatic fat content and insulin resistance in overweight/obese children: a cross-sectional analysis of the Prevention of Diabetes in Kids (PREDIKID) study
}

\author{
Lide Arenaza $^{1 *}$, María Medrano ${ }^{1}$, Maddi Oses ${ }^{1}$, Inge Huybrechts ${ }^{2}$, Ignacio Díez ${ }^{3}$, Hanna Henriksson ${ }^{4,5}$ \\ and Idoia Labayen ${ }^{1}$ \\ ${ }^{1}$ Institute for Innovation and Sustainable Development in Food Chain (IS-FOOD), Public University of Navarra, \\ 31006 Pamplona, Spain \\ ${ }^{2}$ Nutrition and Metabolism Section, International Agency for Research on Cancer, World Health Organization, 69008 Lyon, \\ France \\ ${ }^{3}$ Paediatric Endocrinology Unit, University Hospital of Araba (HUA), 01009 Vitoria-Gasteiz, Spain \\ ${ }^{4}$ PROmoting FITness and Health through physical activity Research Group (PROFITH), Department of Physical and Sports \\ Education, Faculty of Sport Sciences, University of Granada, 18071 Granada, Spain \\ ${ }^{5}$ Department of Medical and Health Sciences, Linköping University, 58183 Linköping, Sweden
}

(Submitted 11 January 2018 - Final revision received 6 September 2018 - Accepted 21 February 2019 - First published online 05 March 2019)

\section{Abstract}

Paediatric non-alcoholic fatty liver disease has increased in parallel with childhood obesity. Dietary habits, particularly products rich in sugars, may influence both hepatic fat and insulin resistance (homeostatic model assessment for insulin resistance (HOMA-IR)). The aim of the study was to examine the association of the consumption of foods and food components, dairy desserts and substitutes (DDS), sugar-sweetened beverages (SSB), as well as total and added sugars, with hepatic fat and HOMA-IR. Dietary intake (two non-consecutive 24h-recalls), hepatic fat (MRI) and HOMA-IR were assessed in 110 overweight/obese children (10.6 (sD 1.1) years old). Linear regression analyses were used to examine the association of dietary intake with hepatic fat and HOMA-IR adjusted for potential confounders (sex, age, energy intake, maternal educational level, total and abdominal adiposity and sugar intake). The results showed that there was a negative association between cereal intake and hepatic fat $(\beta=-0 \cdot 197, P<0 \cdot 05)$. In contrast, both SSB consumption $(\beta=0 \cdot 217 ; P=0.028)$ and sugar in SSB $(\beta=0 \cdot 210, P=0 \cdot 035)$, but not DDS or sugar in DDS or other dietary components, were positively associated with hepatic fat regardless of potential confounders including total sugar intake. In conclusion, cereal intake might decrease hepatic fat, whereas SSB consumption and its sugar content may increase the likelihood of having hepatic steatosis. Although these observations need to be confirmed using experimental evidence, these results suggest that healthy lifestyle intervention programs are needed to improve dietary habits as well as to increase the awareness of the detrimental effects of SSB consumption early in life.

Key words: Hepatic steatosis: Childhood obesity: Homeostatic model assessment for insulin resistance: Dietary intake: Sugar-sweetened beverages

In recent years, the incidence of paediatric non-alcoholic fatty liver disease (NAFLD) has increased worldwide in parallel with childhood obesity rates and, therefore, constitutes an emerging global health concern ${ }^{(1)}$. NAFLD is one of the most common causes of liver disease among children and adolescents ${ }^{(2)}$ and consists of the accumulation of lipids within the hepatocytes in the absence of excess alcohol consumption ${ }^{(3)}$. This pathological disorder is strongly associated with insulin resistance and dyslipidaemia and, therefore, it is considered to be the hepatic manifestation of the metabolic syndrome $e^{(4,5)}$.
Diet and other lifestyle determinants seem to modulate hepatic steatosis development and progression ${ }^{(6)}$. For instance, western dietary habits ${ }^{(7)}$ in both normal weight and overweight/ obese adolescents, as well as energy intake in children ${ }^{(8)}$ or dietary sugar intake such as fructose or sucrose in adults ${ }^{(9)}$, may have an influence on hepatic fat content and other cardiometabolic risk factors ${ }^{(10,11)}$.

Sugar-sweetened beverages (SSB) are the main source of dietary added sugars among children and adolescents, representing around $10-15 \%$ of their dietary energy intake ${ }^{(12,13)}$.

Abbreviations: DDS, dairy desserts and substitutes; HOMA-IR, homeostatic model assessment for insulin resistance; NAFLD, non-alcoholic fatty liver disease; $\mathrm{SSB}$, sugar-sweetened beverages.

* Corresponding author: L. Arenaza, email lide.arenaza@unavarra.es 
Along with the increase in childhood obesity and hepatic steatosis, SSB consumption has also risen in the last years ${ }^{(12)}$. In this context, a systematic review and meta-analysis reported higher risk of NAFLD assessed by abdominal ultrasound or liver biopsy in patients consuming $\mathrm{SSB}^{(14)}$. Other studies have also shown association of SSB consumption with adiposity, type 2 diabetes, the metabolic syndrome and CVD risk factors ${ }^{(15)}$.

Dairy desserts and substitutes (DDS) are other common dietary sugar sources among children since they are often flavoured and sugar sweetened ${ }^{(9,16)}$. Nevertheless, the sugar added in DDS seems not to play such a detrimental role in cardiometabolic health in comparison with sugar from $\mathrm{SSB}^{(17)}$. In contrast, the intake of other food groups and dietary components such as fruits and vegetables ${ }^{(18)}$, dairy products ${ }^{(19)}$ and fibre ${ }^{(20,21)}$ may have a protective role against the metabolic syndrome and hepatic fat accumulation. However, the majority of the studies examining the association of dietary intake with hepatic steatosis are conducted in adolescents ${ }^{(7,18)}$ and adult populations ${ }^{(22)}$. Hence, there are few studies examining the relationship between dietary habits and liver health ${ }^{(23,24)}$ or insulin resistance ${ }^{(25)}$ in overweight and obese children, and, to the best of our knowledge, none of these studies measured hepatic fat content by MRI, which is considered to be the gold standard.

We hypothesised that the intake of products rich in added sugars, such as SSB consumption, would be associated with higher hepatic fat accumulation, whereas no such association would be observed between DDS and hepatic fat content. Likewise, fibre or fibre-rich foods would be beneficial in the prevention of hepatic steatosis and insulin resistance.

Therefore, the aims of the present study were to examine (i) the association of the consumption of dietary foods and components on hepatic fat content and insulin resistance (homeostatic model assessment for insulin resistance (HOMAIR)) and (ii) the influence of SSB and DDS, as well as their sugar content, on hepatic fat content and insulin resistance in overweight/obese children.

\section{Methods}

\section{Study participants and design}

The Prevention of Diabetes in Kids (PREDIKID) study is a randomised controlled trial (ClinicalTrials.gov ID: NCT03027726) examining the effect of a family-based intervention program $^{(26)}$ on diabetes risk of overweight/obese children. The study protocol was approved by the Ethic Committee of Clinical Investigation of Euskadi (PI2014045) and the research was conducted according to the Declaration of Helsinki. Participants were recruited from the Pediatric Endocrinology Unit of the University Hospital of Araba and Primary Care Clinics from Vitoria-Gasteiz and surroundings (North of Spain) in 2017. The main outcome of the trial was insulin resistance ${ }^{(26)}$, whereas the primary outcomes for this sub-study were hepatic fat content and insulin resistance. The inclusion criteria were (1) to be aged between 8 and 12 years, (2) to have overweight or obesity and (3) to meet the international criteria for classification of type 2 diabetes risk. All participants' parents or legal guardians signed an informed written consent for their children in order to be enrolled in the study. The present cross-sectional study includes baseline data of 110 children (10.6 (SD 1.1) years old, 53.1\% girls) with overweight or obesity participating in the PREDIKID study who had dietary intake and hepatic fat content data.

\section{Anthropometry}

Height (SECA 220) and body weight (SECA 760) were measured barefoot according to standard protocols, and waist circumference was measured with a non-elastic tape (SECA 201) following international recommendations. BMI was calculated as body weight divided by height squared (kg/ $\mathrm{m}^{2}$ ) and, thereafter, children were classified as having overweight or obesity according to World Obesity Federation criteria ${ }^{(27)}$. All the anthropometric measurements were carried out by the same trained researcher to avoid interpersonal variability bias. Pubertal stage was directly examined by a paediatrician and classified according to Tanner \& Whitehouse ${ }^{(28)}$ criteria.

\section{Body composition}

Dual-energy X-ray absorptiometry (QDR 4500W; Hologic) was used to evaluate total and abdominal adiposity. Abdominal adiposity was measured by determining three abdominal sections as described elsewhere ${ }^{(29)}$.

\section{Hepatic fat}

Hepatic fat content was measured by MRI (MAGNETOM Avanto; Siemens Healthcare) with phased-array surface coil and a spine array coil equipment. This was provided by the workin-progress software package by Siemens Medical System (version syngo.MR B17A). Briefly, two different three-dimensional gradient-echo sequences in breath-hold and six-echo acquisition with advanced signal analysis were used for hepatic fat quantification and estimation, respectively, as reported elsewhere ${ }^{(30)}$. According to the literature, liver proton density fat fraction estimated by MRI correlates well with histologic steatosis grade in children ${ }^{(31)}$, and therefore MRI is considered a non-invasive and accurate method to detect hepatic steatosis in paediatric population ${ }^{(32)}$.

In accordance with previous studies, the cut-off value of hepatic fat content established for categorising children with and without hepatic steatosis was $5 \cdot 5 \%^{(33,34)}$. Therefore, children with a hepatic fat $\geq 5.5 \%$ were considered as having hepatic steatosis, whereas children with $<5.5 \%$ hepatic fat accumulation as not having hepatic steatosis.

\section{Insulin resistance}

Insulin resistance was determined by calculating HOMA-IR using fasting blood glucose and insulin values with the following formula: (insulin $(\mu \mathrm{U} / \mathrm{ml} \times$ glucose $(\mathrm{mmol} / \mathrm{l})) / 22.5)^{(35)}$. 
Blood sample collection details have been published elsewhere $^{(30)}$.

\section{Dietary intake assessment}

Dietary intake was evaluated, by trained nutritionists, as the mean of two non-consecutive $24 \mathrm{~h}$-recalls during a time span of a week. Children reported, with help of their parents, all the foods and beverages consumed the day before the interview. Pictures of food servings were used to help the participants to estimate food intake. Nutritional composition data was obtained by EasyDiet software. Thereby, energy intake and macro- and micro-nutritional composition data from both interviewed days was obtained. Regarding the food groups, each dietary recall was examined thoroughly, and thereafter, the food consumed was classified into the different food groups so as to obtain the daily food-group intake. Afterwards, the mean consumption of the dietary components consumed in both interviewed days was computed.

Fruits and vegetables were analysed together. Detailed information about foods included in each food category and explanation about each term is available in online Supplementary Table S1. Participants were asked to report product brands, particularly for SSB and DDS, to obtain detailed nutritional composition data, that is, sugar and energy content of each food and beverage. SSB consumption included SSB, while low- or non-energetic beverages were excluded due to the reduction of sugar and replacement for artificial non-energetic sweeteners. DDS were considered milk-derived products with added sugar when its sugar content was $\geq 6 \mathrm{~g} / 100 \mathrm{~g}$ product. The current sugar cut-off value was established based on the natural lactose content of dairy products, assuming that dairy products with higher sugar content may have sugar added in their composition. Also, all dairy product substitutes such as soya-, oat- or almond-beverages were included in DDS, but no cut-off value for sugar content was used since they are not milk-based.

Furthermore, total added sugar was calculated by removing the sugar amount in natural sugar sources such as fruits and dairy products from total dietary sugar intake.

\section{Physical activity}

Accelerometry was used as an objective measurement of total physical activity (wActisleep-BT and wGT3X-BT; ActiGraph). All participants had to wear an accelerometer on the nondominant wrist during a week. Euclidean Norm Minus One was used to quantify the acceleration related to the movement registered expressed in $\mathrm{mg}$ using $\mathrm{R}$ software (version 3.1.2, www.cran.r-project.org) with the GGIR package (version 1.512, https://cran.r-project.org/web/packages/GGIR/).

\section{Statistical analysis}

As the variables did not show a normal distribution, nonparametrical Mann-Whitney $U$ tests were used to examine the differences in continuous variables (i.e. biological characteristics and dietary intake) between children with and without hepatic steatosis, whereas the $\chi^{2}$ test was used for categorical variables. Linear regression analyses were used to examine the association between dietary components (independent variables) and hepatic fat content and insulin resistance (dependent variables). Potential confounders such as sex, age, energy intake and maternal educational level (model 1) and additionally body fat percentage (model 2) or abdominal adiposity (model 3) and dietary simple sugar intake (model 4) were used as covariates in the analyses. Variables with a non-normal distribution were logarithmically transformed for linear regression analyses. Sensitivity analyses were performed in dietary variables and hepatic fat content analysis adjusting additionally for HOMA-IR, physical activity and parental BMI. Collinearity diagnosis tests were also performed between dietary factors to examine whether they were related to one another. Statistical analyses were carried out with the statistical software SPSS version 20.0 (SPSS Inc.) with the significance level of $\alpha=0.05$.

\section{Results}

Biological characteristics and dietary intake of participants with and without hepatic steatosis are shown in Table 1 . The proportion of children with hepatic steatosis was $36.4 \%$. Children with hepatic steatosis had significantly higher BMI $z$-score $(P<0.03)$, waist circumference $(P<0.01)$, total and abdominal adiposity $(P<0 \cdot 01)$, hepatic fat content $(P<0 \cdot 001)$ and HOMAIR $(P<0 \cdot 01)$ compared to children without hepatic steatosis. Likewise, the percentage of children with obesity was higher in the group with hepatic steatosis $(P<0.03)$, while age, sex, high maternal educational level, parental obesity and diabetes and total physical activity were similar between both groups $(P>0 \cdot 05)$, non-Caucasian ethnicity was higher among children with hepatic steatosis $(P<0 \cdot 03)$. In contrast, no significant differences were observed in the intake of macronutrients, simple sugars, added sugars, fibre, cereals, fruits and vegetables, legumes, nuts, dairy products, fish and shellfish and meat and meats products between children with and without hepatic steatosis $(P>0 \cdot 1)$. The consumption of SSB and the sugar content of SSB tended to be higher in children with hepatic steatosis than in their peers without hepatic steatosis $(P<0 \cdot 1)$.

Table 2 shows the associations of dietary components with hepatic fat content and insulin resistance. A negative association was observed between cereal intake and hepatic fat content regardless of sex, age, energy intake and maternal educational level $(P<0 \cdot 02$, model 1$)$. This association was diminished, but remained significant, when both total (model 2 ) and abdominal fat (model 3 ) were entered into the model $(P<0 \cdot 05)$. No statistically significant associations were found between the rest of dietary components and hepatic fat content and insulin resistance in overweight/obese children.

The associations of SSB and DDS intake and the intake of their sugar contents with hepatic fat and HOMA-IR are shown in Table 3. The results showed that both SSB consumption and the intake of sugar from SSB were significantly associated with hepatic fat content regardless of sex, age, energy intake and maternal educational level $(P<0 \cdot 02$, model 1$)$. These relationships were still significant when either body fat percentage or 
Table 1. Biological characteristics and dietary intake of participants with and without hepatic steatosis of the children participating in the Prevention of Diabetes in Kids (PREDIKID) study

(Mean values and standard deviations)

\begin{tabular}{|c|c|c|c|c|c|c|c|}
\hline & \multirow[b]{2}{*}{$n$} & \multicolumn{2}{|c|}{ Children with hepatic steatosis ( $n$ 40) } & \multirow[b]{2}{*}{$n$} & \multicolumn{2}{|c|}{ Children without hepatic steatosis $(n 70)$} & \multirow[b]{2}{*}{$P$} \\
\hline & & Mean & SD & & Mean & SD & \\
\hline Age (years) & 40 & $10 \cdot 5$ & $1 \cdot 1$ & 70 & $10 \cdot 6$ & $1 \cdot 1$ & 0.485 \\
\hline Girls & 40 & & & 70 & & & 0.563 \\
\hline$n$ & & & & & & & \\
\hline$\%$ & & & & & & & \\
\hline BMI z-score & 40 & 0.29 & $1 \cdot 1$ & 70 & -0.16 & 0.9 & $0.028^{*}$ \\
\hline Obese & 40 & & & 70 & & & $0.007^{*}$ \\
\hline$n$ & & & & & & & \\
\hline$\%$ & & & & & & & \\
\hline Waist circumference $(\mathrm{cm})$ & 40 & $82 \cdot 3$ & 7 & 70 & $76 \cdot 7$ & $6 \cdot 6$ & $<0.001^{*}$ \\
\hline Body fat $(\%)$ & 40 & $41 \cdot 2$ & $4 \cdot 2$ & 69 & $38 \cdot 4$ & 4.6 & $0.005^{\star}$ \\
\hline Abdominal fat $(\mathrm{kg})$ & 40 & 3 & $1 \cdot 1$ & 69 & $2 \cdot 3$ & $8 \cdot 4$ & $<0.001^{*}$ \\
\hline Hepatic fat (\%) & 40 & $9 \cdot 2$ & 4.9 & 70 & 3.6 & 1 & $<0.001^{*}$ \\
\hline HOMA-IR & 39 & 3.0 & $1 \cdot 3$ & 69 & $2 \cdot 3$ & 1 & $0.005^{\star}$ \\
\hline High maternal educational level & 39 & & & 70 & & & 0.173 \\
\hline n & & & & & & & \\
\hline$\%$ & & & & & & & \\
\hline Parental obesity & 40 & & & 70 & & & 0.471 \\
\hline$n$ & & & & & & & \\
\hline$\%$ & & & & & & & \\
\hline Parental diabetes & 39 & & & 70 & & & 0.686 \\
\hline$n$ & & & & & & & \\
\hline$\%$ & & & & & & & \\
\hline Non-Caucasian ethnicity & 38 & & & 69 & & & $0.029^{*}$ \\
\hline$n$ & & 10 & & & 7 & & \\
\hline$\%$ & & 25 & & & 10 & & \\
\hline Total physical activity (mg) & 38 & 61.7 & $14 \cdot 6$ & 65 & 65.9 & 17 & 0.286 \\
\hline Energy intake $(\mathrm{kJ} / \mathrm{d})$ & 40 & 7878 & 1498 & 70 & 7460 & 1858 & 0.131 \\
\hline Carbohydrates $(\mathrm{g} / \mathrm{d})$ & 40 & 196 & 60 & 70 & 190 & 50 & 0.737 \\
\hline Fat $(g / d)$ & 40 & 86 & 25 & 70 & 79 & 26 & 0.148 \\
\hline Proteins $(g / d)$ & 40 & 79 & 19 & 70 & 76 & 20 & 0.437 \\
\hline Fibre $(g / d)$ & 40 & 14 & 5 & 70 & 14 & 7 & 0.921 \\
\hline Cereals (g/d) & 40 & 92 & 53 & 70 & 105 & 48 & 0.184 \\
\hline Fruits and vegetables $(\mathrm{g} / \mathrm{d})$ & 40 & 251 & 183 & 70 & 224 & 148 & 0.686 \\
\hline Legumes $(g / d)$ & 40 & $12 \cdot 7$ & $15 \cdot 8$ & 70 & $14 \cdot 3$ & $20 \cdot 8$ & 0.799 \\
\hline Nuts $(g / d)$ & 40 & 2 & 6.5 & 70 & 3 & 9 & 0.697 \\
\hline Dairy products $(\mathrm{g} / \mathrm{d})$ & 40 & 347 & 188 & 70 & 321 & 134 & 0.725 \\
\hline Fish and shellfish $(\mathrm{g} / \mathrm{d})$ & 40 & 47 & 57 & 70 & 34 & 45 & 0.260 \\
\hline Meat and meat products $(\mathrm{g} / \mathrm{d})$ & 40 & 107 & 70 & 70 & 98 & 76 & 0.389 \\
\hline Simple sugars $(g / d)$ & 40 & 89 & 37 & 70 & 82 & 26 & 0.445 \\
\hline Added sugar (g/d) & 40 & 60 & 31 & 70 & 51 & 22 & 0.146 \\
\hline $\mathrm{SSB}(\mathrm{ml} / \mathrm{d})$ & 40 & 117 & 169 & 70 & 53 & 91 & 0.069 \\
\hline Sugar from SSB $(g / d)$ & 40 & 9.8 & $14 \cdot 7$ & 70 & 4.6 & 8.0 & 0.094 \\
\hline $\operatorname{DDS}(\mathrm{g} / \mathrm{d})$ & 40 & 81 & 77 & 70 & 85 & 93 & 0.839 \\
\hline Sugar from DDS $(\mathrm{g} / \mathrm{d})$ & 40 & $10 \cdot 6$ & 10 & 70 & $10 \cdot 8$ & $12 \cdot 0$ & 0.739 \\
\hline
\end{tabular}

HOMA-IR, homeostatic model assessment for insulin resistance; SSB, sugar-sweetened beverages; DDS, dairy desserts and substitutes.

${ }^{*} P<0.05$.

abdominal fat were included into the model $(P<0 \cdot 02$, models 2 and 3). Interestingly, the association of SSB consumption and the intake of sugar from SSB with hepatic fat content remained statistically significant even after further adjustment for total simple sugar intake $(P<0 \cdot 05$, model 4$)$. In a sensitivity (additional adjustment) analysis with insulin resistance $(\beta=0 \cdot 212$, $P=0.036$ and $\beta=0.204, P=0.045$ for SSB and sugar from SSB, respectively), physical activity $(\beta=0.217, \quad P=0.032$ and $\beta=0.206, P=0.043$ for SSB and sugar from SSB, respectively) and parental BMI $(\beta=0.207, P=0.037$ and $\beta=0.199, P=0.047$ for SSB and sugar from SSB, respectively) this association remained statistically significant. Continuous logistic regression was performed to analyse the effect of SSB consumption in children with and without hepatic steatosis (sex, age, maternal educational level and energy intake adjustments); and even though it was not statistically significant, a trend to signification was observed (OR 1.003, $95 \%$ CI 1.000, 1.007, $P=0.051$ ). On the contrary, no significant relationships were observed between DDS consumption and sugar from DDS with hepatic fat content in overweight/obese children $(P>0 \cdot 05)$. Neither SSB nor DDS consumption were associated with insulin resistance $(P>0 \cdot 05)$. No collinearity was found between dietary factors.

\section{Discussion}

The present study examined the influence of several food groups and dietary components on hepatic fat content and 
Table 2. Association of dietary energy and macronutrient intake, and other dietary components with hepatic fat content and insulin resistance in overweight/ obese children*

\begin{tabular}{|c|c|c|c|c|c|c|c|c|c|c|c|c|}
\hline & \multicolumn{6}{|c|}{ Hepatic fat content (\%) } & \multicolumn{6}{|c|}{ HOMA-IR } \\
\hline & \multicolumn{2}{|c|}{ Model 1} & \multicolumn{2}{|c|}{ Model 2} & \multicolumn{2}{|c|}{ Model 3} & \multicolumn{2}{|c|}{ Model 1} & \multicolumn{2}{|c|}{ Model 2} & \multicolumn{2}{|c|}{ Model 3} \\
\hline & $\beta$ & $P$ & $\beta$ & $P$ & $\beta$ & $P$ & $\beta$ & $P$ & $\beta$ & $P$ & $\beta$ & $P$ \\
\hline Energy $(\mathrm{kJ} / \mathrm{d})$ & 0.033 & 0.740 & - & - & - & - & 0.068 & 0.487 & - & - & - & - \\
\hline Carbohydrates (g/d) & -0.016 & 0.919 & 0.017 & 0.913 & 0.007 & 0.965 & 0.048 & 0.761 & 0.112 & 0.452 & 0.098 & 0.477 \\
\hline Fat $(g / d)$ & 0.034 & 0.838 & 0.041 & 0.796 & 0.044 & 0.779 & -0.026 & 0.874 & -0.063 & 0.693 & -0.064 & 0.665 \\
\hline Proteins (g/d) & -0.017 & 0.907 & -0.097 & 0.477 & -0.084 & 0.533 & -0.041 & 0.772 & -0.092 & 0.485 & -0.066 & 0.591 \\
\hline Fibre $(\mathrm{g} / \mathrm{d})$ & -0.118 & 0.261 & -0.152 & 0.132 & -0.157 & 0.114 & 0.052 & 0.620 & 0.037 & 0.706 & 0.028 & 0.763 \\
\hline Cereals $(g / d)$ & -0.254 & $0.013 \dagger$ & -0.211 & $0.036 \dagger$ & -0.197 & $0.048 \dagger$ & 0.003 & 0.979 & 0.062 & 0.530 & 0.091 & 0.328 \\
\hline Fruits and vegetables $(\mathrm{g} / \mathrm{d})$ & 0.028 & 0.771 & 0.044 & 0.638 & 0.062 & 0.501 & 0.063 & 0.511 & 0.080 & 0.381 & 0.116 & 0.173 \\
\hline Dairy products (g/d) & 0.085 & 0.384 & 0.125 & 0.190 & 0.095 & 0.322 & -0.073 & 0.454 & -0.018 & 0.849 & -0.085 & 0.334 \\
\hline Fish and shellfish $(\mathrm{g} / \mathrm{d})$ & 0.152 & 0.114 & 0.112 & 0.230 & 0.103 & 0.263 & 0.110 & 0.250 & 0.079 & 0.389 & 0.069 & 0.422 \\
\hline Meat and meat products $(\mathrm{g} / \mathrm{d})$ & -0.007 & 0.949 & -0.008 & 0.939 & 0.001 & 0.995 & -0.076 & 0.492 & -0.134 & 0.207 & -0.119 & 0.230 \\
\hline
\end{tabular}

HOMA-IR, homeostatic model assessment for insulin resistance.

* Model 1: adjusted for sex, age, energy intake and maternal educational level; model 2: model 1 additionally adjusted for body fat percentage; model 3 : model 2 additionally adjusted for abdominal adiposity. $\dagger P<0.05$.

Table 3. Associations of sugar-sweetened beverages (SSB) and dairy desserts and substitutes (DDS) consumption as well as their sugar content with hepatic fat content and insulin resistance (homeostatic model assessment for insulin resistance (HOMA-IR)) in overweight/obese children*

\begin{tabular}{|c|c|c|c|c|c|c|c|c|c|c|c|c|c|c|c|c|}
\hline & \multicolumn{8}{|c|}{ Hepatic fat content $(\%)$} & \multicolumn{8}{|c|}{ HOMA-IR } \\
\hline & \multicolumn{2}{|c|}{ Model 1} & \multicolumn{2}{|c|}{ Model 2} & \multicolumn{2}{|c|}{ Model 3} & \multicolumn{2}{|c|}{ Model 4} & \multicolumn{2}{|c|}{ Model 1} & \multicolumn{2}{|c|}{ Model 2} & \multicolumn{2}{|c|}{ Model 3} & \multicolumn{2}{|c|}{ Model 4} \\
\hline & $\beta$ & $P$ & $\beta$ & $P$ & $\beta$ & $P$ & $\beta$ & $P$ & $\beta$ & $P$ & $\beta$ & $P$ & $\beta$ & $P$ & $\beta$ & $P$ \\
\hline SSB (g/d) & 0.263 & $0.008 \dagger$ & 0.250 & $0.009 \dagger$ & 0.243 & $0.010 \dagger$ & 0.217 & $0.028 \dagger$ & 0.108 & 0.276 & 0.102 & 0.282 & 0.085 & 0.336 & 0.094 & 0.318 \\
\hline Sugar from SSB $(\mathrm{g} / \mathrm{d})$ & 0.254 & $0.011 \dagger$ & 0.243 & $0.011 \dagger$ & 0.237 & $0.012 \dagger$ & 0.210 & $0.035 \dagger$ & 0.106 & 0.292 & 0.100 & 0.298 & 0.085 & 0.340 & 0.094 & 0.322 \\
\hline $\operatorname{DDS}(\mathrm{g} / \mathrm{d})$ & -0.051 & 0.612 & -0.067 & 0.498 & -0.048 & 0.621 & -0.081 & 0.412 & -0.054 & 0.585 & $-0 \cdot 128$ & 0.184 & -0.092 & 0.309 & -0.096 & 0.299 \\
\hline Sugar from DDS $(g / d)$ & -0.037 & 0.715 & -0.053 & 0.593 & -0.036 & 0.715 & -0.070 & 0.483 & -0.061 & 0.540 & -0.135 & 0.163 & -0.099 & 0.274 & -0.104 & 0.263 \\
\hline Simple sugar $(g / d)$ & 0.131 & 0.270 & 0.171 & 0.135 & 0.179 & 0.112 & - & - & -0.053 & 0.648 & -0.013 & 0.904 & 0.004 & 0.969 & - & - \\
\hline Added sugar (g/d) & 0.209 & 0.074 & 0.195 & 0.084 & 0.208 & 0.061 & 0.178 & 0.314 & 0.005 & 0.964 & -0.024 & 0.827 & 0.002 & 0.986 & -0.003 & 0.983 \\
\hline
\end{tabular}

* Model 1: adjusted for sex, age, energy intake and maternal education level; model 2: model 1 additionally adjusted for body fat percentage; model 3: model 2 additionally adjusted for abdominal adiposity; model 4: model 3 additionally adjusted for simple sugar intake. $\dagger P<0.05$.

insulin resistance among pre-adolescent overweight/obese children. The main findings were that cereal intake was negatively associated with hepatic fat content but not with insulin resistance, whereas the rest of the food groups and macronutrients were associated neither with hepatic fat content nor with insulin resistance. Moreover, SSB consumption and sugar from SSB were positively associated with hepatic fat accumulation, but not with insulin resistance. In contrast, we did not observe any significant relationships between DDS and hepatic fat content or insulin resistance among overweight/obese children aged 8-12 years.

Almost half of the children in the study $(36.4 \%$ of the participants) presented with hepatic steatosis. It is well-known that excess adiposity increases the risk of having NAFLD ${ }^{(36)}$. Our findings about prevalence are in agreement with those from two previous studies. One of them reported that whilst the prevalence of paediatric hepatic steatosis is lower than $10 \%$ in general population, the prevalence among children with obesity can reach $80 \%{ }^{(37)}$. According to other study, the prevalence of NAFLD in children with normal weight was $2.6 \%$, which increased to $20-77 \%$ among obese children and adolescents ${ }^{(38)}$. Nevertheless, it should be pointed out that the range of prevalence might vary depending on the diagnosis method used for measuring hepatic fat content. In this study, hepatic fat content was measured by MRI, which is a more accurate method compared to ultrasound, and in fact, it has been considered a non-invasive appropriate alternative for paediatric population ${ }^{(33)}$. Furthermore, and in line with other studies $^{(34)}$, ethnic differences between Hispanic, Black and White population were observed in the prevalence of hepatic steatosis. Regarding body composition, children with hepatic steatosis had higher values of waist circumference as well as total and abdominal adiposity compared to children without hepatic steatosis. On the contrary, participants with hepatic steatosis did not show higher values of BMI, which demonstrates the limitations of using BMI as a proxy of body fatness in children ${ }^{(39)}$. Accordingly, based on the strong association between abdominal adiposity and hepatic steatosis, Alisi et al. ${ }^{(40)}$ reported that abdominal obesity is more powerful than BMI in predicting hepatic steatosis.

Overall, we found only few differences in dietary intake variables between children with and without hepatic steatosis. In contrast to our results, a previous study reported that adolescents with hepatic steatosis had higher intake of total fat and 
fried foods compared to their peers without hepatic steatosis ${ }^{(41)}$. However, in the present study children with hepatic steatosis tended to consume more SSB compared to children without hepatic steatosis, which is consistent with the association between SSB consumption and NAFLD ${ }^{(10)}$. Our observations suggest that cereal intake may be protective against hepatic steatosis development. On the contrary, Georgoulis et al. ${ }^{(42)}$ reported that refined grains were associated with higher likelihood of having NAFLD in adults, whereas whole grain consumption favourably affected clinical characteristics of participants with NAFLD as a result of being rich in fibre and having lower glycaemic index. Unfortunately, due to the infrequent intake of whole grains by children from the PREDIKID study, we could not examine the influence of whole cereals on hepatic health. On the other hand, in disagreement with other studies in which whole cereal intake was inversely associated with insulin resistance among adolescents ${ }^{(43)}$, no association was found between cereal intake and HOMA-IR in overweight/obese children.

We observed that 39.8 and $65.5 \%$ of the children consumed SSB and DDS, respectively (data not shown). Moreover, we found a positive association between SSB consumption and sugar in SSB and hepatic fat content regardless of sex, age, energy intake, maternal educational level, body fat percentage, abdominal obesity and total simple sugar intake. Of note, while in the USA SSB are primarily sweetened with high fructose maize syrup, in Europe sucrose is commonly used as the main sweetener $^{(44)}$, which is a disaccharide composed of fructose and glucose. Although several studies report the association of both fructose $^{(45)}$ and SSB consumption ${ }^{(46,47)}$ with adiposity and cardiometabolic risk factors in adults and adolescents, few studies focused on children to date. Furthermore, to the best of our knowledge, this is the first study examining the influence of SSB consumption on hepatic fat content in overweight/obese children. Interestingly, neither SSB nor sugar from SSB was associated with insulin resistance. In addition, based on the fact that impaired insulin signalling originated by dysfunctional adipose tissue induces insulin resistance, insulin resistance might be a secondary effect of excess hepatic fat. Despite the lack of the association in our study, other authors reported that daily intake of SSB was associated with increased HOMA-IR in adolescents $^{(48)}$. The possible mechanism of how fructose can induce NAFLD has largely been studied in animal models. Briefly, fructose is absorbed from the intestine into the portal vein and goes directly to the liver, where it stimulates de novo lipogenesis by promoting hepatic lipid accumulation ${ }^{(40,46)}$, which may explain NAFLD development. Nonetheless, not only fructose but also sucrose-containing beverages have been shown to increase hepatic fat as well as visceral and muscle fat and $\mathrm{TAG}^{(49)}$

In contrast, DDS consumption was not significantly associated with hepatic fat content and insulin resistance in our sample of overweight/obese children. This finding is in line with previous studies suggesting that dairy fat intake as well as other nutrients like Ca (which are mostly present in DDS, but not in SSB) could be protective against hepatic fat accumulation in adults ${ }^{(50)}$ and abdominal adiposity in female adolescents ${ }^{(51)}$. In addition, the fact that DDS are commonly consumed with a meal, whereas people tend to drink SSB between meals, could also explain the influence on these outcomes. Accordingly, previous studies reported that snacking promotes liver fat accumulation $^{(52,53)}$. As a consequence of the rise of SSB consumption in children, initiatives such as the implementation of SSB-associated tax in several countries have been carried out to prevent obesity and related comorbidities. The purchases of SSB decreased after Mexico implemented a tax, so that this seems to be a successful intervention from global policies ${ }^{(54)}$.

\section{Strengths and limitations}

The use of MRI to measure hepatic fat content should be considered as study strength for its accuracy, while ultrasonography is used in most of the other studies. Moreover, detailed nutritional composition information of SSB and DDS was obtained by analysing the labels of products brands. However, the current study also has some limitations. These findings should be analysed carefully due to its cross-sectional design and, therefore, prospective studies are needed since experimental evidence would strengthen these cross-section observations.

\section{Conclusions}

The main findings in the present study suggest that cereal intake seems to be associated with lower liver fat content, whereas SSB consumption and sugar from SSB might increase hepatic fat content among overweight/obese children. In contrast, neither DDS consumption nor sugar from DDS seems to modulate cardiometabolic risk factors, and last but not least, no association was found between dietary intake and insulin resistance in children with excess adiposity. Our results suggest that healthy lifestyle intervention programs are needed to improve dietary habits as well as to increase the awareness of the detrimental effects of SSB consumption early in life.

\section{Acknowledgements}

The authors acknowledge all the children and their parents or legal guardians for participating in the study. The authors also thank the paediatricians for their collaboration in recruiting participants and to the pre- and post-graduate students for their collaboration in both data collection and intervention program sessions.

This project was supported by the Spanish Ministry of Industry and Competitiveness (DEP2016-78377-R), by 'Fondos Estructurales de la Unión Europea (FEDER), Una manera de hacer Europa' and by the University of the Basque Country (GIU14/21). This work was also supported by grants from Spanish Ministry of Economy and Competitiveness (RYC 201005957; RYC-2011-09011), by the Education Department of the Government of the Basque Country (PRE_2016_1_0057, PRE_2017_2_0224 and PRE_2018_2_0057), by the Spanish Ministry of Education, Culture and Sports (FPU14/03329), and by the Swedish Society of Medicine.

I. L. conceived and designed the study, L. A. and I. L. drafted the manuscript, L. A., M. M., M. O. and I. L. collected the data and L. A., M. M., M. O., I. H., I. D., H. H. and I. L. were involved 
in the interpretation of the results. All the authors critically revised the manuscript for important intellectual content and approved the final version of the submitted manuscript.

The authors declare that there are no conflicts of interest.

\section{Supplementary material}

For supplementary material/s referred to in this article, please visit https://doi.org/10.1017/S0007114519000436

\section{References}

1. Than NN \& Newsome PN (2015) A concise review of nonalcoholic fatty liver disease. Atherosclerosis 239, 192-202.

2. Yang M, Gong S, Ye SQ, et al. (2014) Non-alcoholic fatty liver disease in children: focus on nutritional interventions. Nutrients 6, 4691-4705.

3. Abenavoli L, Milic N, Peta V, et al. (2014) Alimentary regimen in non-alcoholic fatty liver disease: Mediterranean diet. World J Gastroenterol 20, 16831-16840.

4. Ferolla SM, Silva LC, De Lourdes M, et al. (2015) Dietary approach in the treatment of nonalcoholic fatty liver disease. World J Hepatol 7, 2522-2534.

5. Alkhater SA (2015) Paediatric non-alcoholic fatty liver disease: an overview. Obes Rev 16, 393-405.

6. Zivkovic AM, German JB \& Sanyal AJ (2007) Comparative review of diets for the metabolic syndrome: implications for nonalcoholic fatty liver disease. Am J Clin Nutr 86, 285-300.

7. Oddy WH, Herbison CE, Jacoby P, et al. (2013) The Western dietary pattern is prospectively associated with nonalcoholic fatty liver disease in adolescence. Am J Gastroenterol 108, $778-785$.

8. Anderson EL, Howe LD, Fraser A, et al. (2015) Childhood energy intake is associated with nonalcoholic fatty liver disease in adolescents. J Nutr 145, 983-989.

9. Azaïs-Braesco V, Sluik D, Maillot M, et al. (2017) A review of total \& added sugar intakes and dietary sources in Europe. Nutr J 16, 6.

10. Ma J, Fox CS, Jacques PF, et al. (2015) Sugar-sweetened beverage, diet soda, and fatty liver disease in the Framingham Heart Study cohorts. J Hepatol 63, 462-469.

11. Te Morenga LA, Howatson AJ, Jones RM, et al. (2014) Dietary sugars and cardiometabolic risk: systematic review and meta-analyses of randomized controlled trials of the effects on blood pressure and lipids. Am J Clin Nutr 100, 65-79.

12. Keller A \& Bucher Della Torre S (2015) Sugar-sweetened beverages and obesity among children and adolescents: a review of systematic literature. Child Obes 11, 338-346.

13. Hu FB \& Malik VS (2011) Sugar-sweetened beverages and risk of obesity and type 2 diabetes: epidemiologic evidence. Physiol Behav 100, 47-54.

14. Wijarnpreecha K, Thongprayoon C, Edmonds PJ, et al. (2016) Associations of sugar- and artificially sweetened soda with nonalcoholic fatty liver disease: a systematic review and metaanalysis. QJM 109, 461-466.

15. Jensen BW, Nielsen BM, Husby I, et al. (2013) Association between sweet drink intake and adiposity in Danish children participating in a long-term intervention study. Pediatr Obes $\mathbf{8}$, 259-270.

16. Stern D, Piernas C, Barquera S, et al. (2014) Caloric beverages were major sources of energy among children and adults in Mexico. J Nutr 144, 949-956.
17. Khan TA \& Sievenpiper JL (2016) Controversies about sugars: results from systematic reviews and meta analyses on obesity, cardiometabolic disease and diabetes. Eur J Nutr 55, 25-43.

18. Cook LT, O'Reilly GA, Goran MI, et al. (2014) Vegetable consumption is linked to decreased visceral and liver fat and improved insulin resistance in overweight Latino youth. J Acad Nutr Diet 114, 1776-1783.

19. Shin H, Yoon YS, Lee Y, et al. (2013) Dairy product intake is inversely associated with metabolic syndrome in Korean adults: Anseong and Ansan cohort of the Korean genome and epidemiology study. J Korean Med Sci 28, 1482-1488.

20. Cantero I, Abete I, Monreal JI, et al. (2017) Fruit fiber consumption specifically improves liver health status in obese subjects under energy restriction. Nutrients $\mathbf{9}, 667$.

21. Davis J, Alexander K \& Ventura E (2009) Inverse relation between dietary fiber intake and visceral adiposity in overweight Latino youth. Am J Clin Nutr 90, 1160-1166.

22. Jia Q, Xia Y, Zhang Q, et al. (2015) Dietary patterns are associated with prevalence of fatty liver disease in adults. EurJ Clin Nutr 69, 914-921.

23. Papandreou D, Karabouta Z, Pantoleon A, et al. (2012) Investigation of anthropometric, biochemical and dietary parameters of obese children with and without non-alcoholic fatty liver disease. Appetite 59, 939-944.

24. Félix DR, Costenaro F, Gottschall CBA, et al. (2016) Nonalcoholic fatty liver disease (NAFLD) in obese children- effect of refined carbohydrates in diet. BMC Pediatr 16, 187.

25. Visuthranukul C, Sirimongkol P, Prachansuwan A, et al. (2015) Low-glycemic index diet may improve insulin sensitivity in obese children. Pediatr Res 78, 567-573.

26. Arenaza L, Medrano M, Amasene M, et al. (2017) Prevention of diabetes in overweight/obese children through a family based intervention program including supervised exercise (PREDIKID project): study protocol for a randomized controlled trial. Trials 18, 372 .

27. Cole TJ \& Lobstein T (2012) Extended international (IOTF) body mass index cut-offs for thinness, overweight and obesity. Pediatr Obes 7, 284-294.

28. Tanner JM \& Whitehouse RH (1976) Clinical longitudinal standards for height, weight, height velocity, weight velocity, and stages of puberty. Arch Dis Child 51, 170-179.

29. Labayen I, Ruiz JR, Vicente-Rodríguez G, et al. (2009) Early life programming of abdominal adiposity in adolescents: the HELENA. Diabetes Care 32, 2120-2122.

30. Medrano M, Maiz E, Maldonado-Martin S, et al. (2015) The effect of a multidisciplinary intervention program on hepatic adiposity in overweight-obese children: protocol of the EFIGRO study. Contemp Clin Trials 45, 346-355.

31. Schwimmer JB, Middleton MS, Behling C, et al. (2015) Magnetic resonance imaging and liver histology as biomarkers of hepatic steatosis in children with nonalcoholic fatty liver disease. Hepatology 61, 1887-1895.

32. Hsu E \& Murray K (2012) Is nonalcoholic fatty liver disease in children the same disease as in adults? Clin Liver Dis 16, 587-598.

33. Younossi ZM, Loomba R, Rinella ME, et al. (2018) Current and future therapeutic regimens for nonalcoholic fatty liver disease (NAFLD) and nonalcoholic steatohepatitis. Hepatology $6 \mathbf{8}$, 361-371.

34. Browning JD, Szczepaniak LS, Dobbins R, et al. (2004) Prevalence of hepatic steatosis in an urban population in the United States: impact of ethnicity. Hepatology 40, 1387-1395.

35. Matthews D, Hosker J \& Rudenski A (1985) Homeostasis model assessment: insulin resistance and beta-cell function from fasting plasma glucose and insulin concentrations in man. Diabetologia 28, 412-419. 
36. Barshop NJ, Sirlin CB, Schwimmer JB, et al. (2008) Review article: epidemiology, pathogenesis and potential treatments of paediatric non-alcoholic fatty liver disease. Aliment Pharmacol Ther 28, 13-24.

37. Alisi A, Feldstein AE, Villani A, et al. (2012) Pediatric nonalcoholic fatty liver disease: a multidisciplinary approach. Nat Rev Gastroenterol Hepatol 9, 152-161.

38. Reinehr T, Schmidt C, Toschke AM, et al. (2009) Lifestyle intervention in obese children with non- alcoholic fatty liver disease: 2-year follow-up study. Arch Dis Child 94, 437-442.

39. Freedman DS, Sherry B, et al. (2009) The validity of BMI as an indicator of body fatness and risk among children. Pediatrics 124, S23-S34.

40. Alisi A, Manco M, Vania A, et al. (2009) Pediatric nonalcoholic fatty liver disease. J Pediatr 55, 469-474.

41. Macintosh AC, Hay J, Wicklow BA, et al. (2014) Dietary determinants of hepatic steatosis and visceral adiposity in overweight and obese youth at risk of type 2 diabetes. $A m \mathrm{~J}$ Clin Nutr 99, 804-812.

42. Georgoulis M, Kontogianni MD \& Tileli N (2014) The impact of cereal grain consumption on the development and severity of non-alcoholic fatty liver disease. Eur J Nutr $\mathbf{5 3}$, $1727-1735$.

43. Steffen LM, Jacobs DR, Murtaugh MA, et al. (2003) Whole grain intake is associated with lower body mass and greater insulin sensitivity among adolescents. Am J Epidemiol 158, $243-250$

44. Malik VS \& Hu FB (2015) Fructose and cardiometabolic health: what the evidence from sugar-sweetened beverages tells us. J Am Coll Cardiol 66, 1615-1624.

45. Alwahsh SM \& Gebhardt R (2017) Dietary fructose as a risk factor for non-alcoholic fatty liver disease (NAFLD). Arch Toxicol 91, 1545-1563.
46. Wang J, Light K, Loughlin JO, et al. (2014) Consumption of added sugars from liquid but not solid sources predicts impaired glucose homeostasis and insulin resistance among youth at risk of obesity. J Nutr 144, 81-86.

47. Chan T, Lin W, Huang H, et al. (2014) Consumption of sugarsweetened beverages is associated with components of the metabolic syndrome in adolescents. Nutrients 6, 2088-2103.

48. Kondaki K, Grammatikaki E, Jime D, et al. (2012) Daily sugarsweetened beverage consumption and insulin resistance in European adolescents: the HELENA (Healthy Lifestyle in Europe by Nutrition in Adolescence) Study. Public Health Nutr 16, 479-486.

49. Maersk M, Belza A, Stodkilde-jorgensen H, et al. (2012) Sucrose-sweetened beverages increase fat storage in the liver, muscle, and visceral fat depot: a 6-mo randomized intervention study. Am J Clin Nutr 95, 283-289.

50. Kratz M, Marcovina S, Nelson JE, et al. (2014) Dairy fat intake is associated with glucose tolerance, hepatic and systemic insulin sensitivity and liver fat but not b 412-cell function in humans. Am J Clin Nutr 99, 1385-1396.

51. Castro Burbano J, Fajardo Vanegas P, et al. (2016) Relationship between dietary calcium intake and adiposity in female adolescents. Endocrinol Nutr 63, 58-63.

52. Piernas C \& Popkin B (2010) Trends in snacking among U.S. children. Heal Aff 29, 398-404.

53. Koopman KE, Caan MWA, Nederveen AJ, et al. (2014) Hypercaloric diets with increased meal frequency, but not meal size, increase intrahepatic triglycerides: a randomized controlled trial. Hepatology 60, 545-553.

54. Colchero MA, Molina M \& Guerrero-l CM (2017) After Mexico implemented a tax, purchases of sugar-sweetened beverages decreased and water increased: difference by place of residence, household composition, and income level. J Nutr 147, $1552-1557$. 